\title{
THE SINGULAR HOMOGENEOUS SOLUTIONS TO ONE PHASE FREE BOUNDARY PROBLEM
}

\author{
GUANGHAO HONG \\ (Communicated by Tatiana Toro)
}

\begin{abstract}
We provide some new examples of singular homogeneous of degree one solutions to the well-known one phase free boundary problem. They are critical points of the functional $J(v, B)=\int_{B}|\nabla v|^{2}+\chi_{\{v>0\}}$. We also discuss their stability using a criteria of Caffarelli, Jerison and Kenig.
\end{abstract}

\section{INTRODUCTION}

We consider the variational problem of the functional

$$
J(v, D)=\int_{D}|\nabla v|^{2}+\chi_{\{v>0\}}
$$

in the convex set

$$
K:=\left\{v \in L_{l o c}^{1}(D) \mid \nabla v \in L^{2}(D) \text { and } v=u^{0} \geq 0 \text { on a essential part of } \partial D\right\} .
$$

A non-negative critical point $u$ satisfies the following equation in a weak sense:

$$
\left\{\begin{array}{cll}
\triangle u=0 & \text { in } \quad\{u>0\} \cap D, \\
\frac{\partial u}{\partial \nu}=-1 & \text { on } \quad \partial\{u>0\} \cap D .
\end{array}\right.
$$

In 1981, Alt and Caffarelli [1] proved that for a local minimum $u$ the reduced free boundary $\partial_{\text {red }}\{u>0\}$ is a regular surface, and in 2 dimensions the singular points cannot occur. They gave an example in 3 dimensions which has an isolated singular point on the free boundary. They showed that this example is a critical point but not a local minimum.

In 1999, Weiss 4] proved that the existence of singularities is equivalent to the existence of an absolute minimum $u^{*}$ such that $u^{*}$ is homogeneous of degree 1 with one and only one singularity at 0 . This leads to the following partial regularity: there is a maximal dimensions $k^{*} \geq 3$ such that for $n<k^{*}$ the free boundary must be smooth, for $n=k^{*}$ there can be isolated singular points, and for $n>k^{*}$ the Hausdorff dimensions of singular set is less than $n-k^{*}$.

In 2004, Caffareli, Jerison and Kenig [2] proved that in 3 dimensions all singular homogeneous of degree 1 critical points are unstable, and this implied $k^{*} \geq 4$. They also showed that the simplest axis-symmetric singular solutions (the higher dimensional generalization of the above mentioned Alt-Caffarelli example in 3 dimensions) are unstable in dimensions 3-6. Later, De Silva and Jerison [3] proved

Received by the editors August 24, 2013 and, in revised form, May 31, 2014.

2010 Mathematics Subject Classification. Primary 35J25, 35B65; Secondary 35J05.

Key words and phrases. Free boundary, symmetry, regularity, stability. 
that this solution is an absolute minimum in dimension 7 , and this implied $k^{*} \leq 7$. One guessed $k^{*}=7$, but there has not been any further progress since then.

In this paper, we construct some new singular homogeneous of degree 1 critical points to the one phase free boundary problem, which include the known axissymmetric solution as a special case. We have 8 new solutions in dimensions 4,5 and 6 , all of them are unstable. In 7 dimension, we have a new solution that satisfies the necessary condition for stability in [2] and is likely to be a global minimizer.

Definition 1.1. We say $u \in C\left(\mathbb{R}^{n}\right)$ is a homogeneous solution to the one phase free boundary problem if: $u \geq 0 ; u(r x)=r u(x) ; u$ is harmonic in $\Gamma:=\{u>0\}$; $\partial \Gamma \backslash\{0\}$ is smooth and $\frac{\partial u}{\partial \nu}=-1$ on $\partial \Gamma \backslash\{0\}$, where $\nu$ is the outer unit normal vector of $\Gamma$. We say $u$ is singular if $u(x) \neq x_{1}^{+}$(a module rotation).

Such a $u$ is determined by its values on the unit sphere $S^{n-1}$ as a result of homogeneity. On $S^{n-1}, \triangle u=u_{r r}+\frac{n-1}{r} u_{r}+\triangle_{S^{n-1}} u=\triangle_{S^{n-1}} u+(n-1) u$. So $\left.u\right|_{S^{n-1}}$ (we still denote it by $u$ ) satisfies the following:

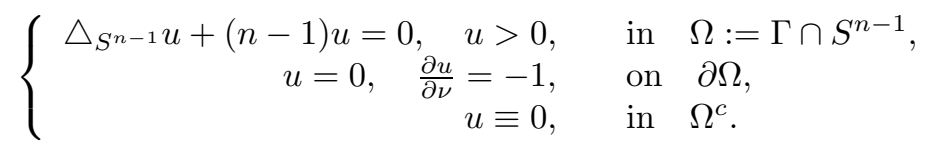

Therefore, to construct a homogeneous solution to one phase free boundary problem, it is equivalent to find a solution to the overdetermined first eigenvalue problem (1.1) in $S^{n-1}$.

From now on, we consider $u$ as a function in $S^{n-1}$. Denote $\operatorname{Ridge}(u):=\{x \in$ $\left.\Omega: u(x)=\max _{y \in \Omega} u(y)\right\}$. We will construct some special solutions $u$ whose values $u(x)$ only depend on $\operatorname{dist}_{S^{n-1}}(x, \operatorname{Ridge}(u))$, the intrinsic distance from $x$ to the set $\operatorname{Ridge}(u)$ in $S^{n-1}$. The ridges in our examples are

$$
S^{p}(1):=\left\{x \in \mathbb{R}^{n}: x_{1}^{2}+\cdots+x_{p+1}^{2}=1, x_{p+2}=\cdots=x_{n}=0\right\}
$$

for $p=1, \cdots, n-2$. Note that when $p=n-2, u$ is the known axis-symmetric solution. If $\operatorname{Ridge}(u)=\{(1,0, \cdots, 0)\}, u$ is the regular half space solution. In the case that $n$ is even, we also have a solution $u$ that has the Clifford torus

$$
S^{\frac{n-2}{2}}\left(\frac{1}{\sqrt{2}}\right) \times S^{\frac{n-2}{2}}\left(\frac{1}{\sqrt{2}}\right):=\left\{x \in \mathbb{R}^{n}: x_{1}^{2}+\cdots+x_{\frac{n}{2}}^{2}=x_{\frac{n}{2}+1}^{2}+\cdots+x_{n}^{2}=\frac{1}{2}\right\}
$$

as its ridge.

\section{Construction of homogeneous solutions}

We first prove a lemma.

Lemma 2.1. Assume that $h(t) \in C^{2}(0, T) \cap C^{1}[0, T)$ solves the problem

$$
\left\{\begin{aligned}
\left(a(t) h^{\prime}(t)\right)^{\prime}+b(t) h(t) & =0, \quad t \in(0, T), \\
h(0)=1, h^{\prime}(0) & =0,
\end{aligned}\right.
$$

where $a(t)>0, b(t)>0$ for $t \in(0, T)$. If $\limsup _{t \rightarrow 0}|a(t)|<+\infty$ and for some $N \geq 1 \lim _{t \rightarrow T} \frac{a(t)}{(T-t)^{N}}=k>0$, then there exists $t_{0} \in(0, T)$ such that $h\left(t_{0}\right)=0$, $h^{\prime}\left(t_{0}\right)<0$ and $h(t)>0$ for $t \in\left(0, t_{0}\right)$. 
Proof. Suppose $h(t)>0$ for $t \in[0, T)$. Integrating the equation from $\epsilon^{\prime}$ to $T-\epsilon$ and letting $\epsilon^{\prime}$ go to 0 , we get

$$
a(T-\epsilon) h^{\prime}(T-\epsilon)=-\int_{0}^{T-\epsilon} b(t) h(t) d t \leq-c_{1}<0 .
$$

There exists $\epsilon_{0}>0$ such that

$$
1.1 k^{N} \epsilon \leq a(T-\epsilon) \leq 0.9 k^{N} \epsilon \text { for } \epsilon \in\left(0, \epsilon_{0}\right) .
$$

So

$$
h^{\prime}(T-\epsilon) \leq \frac{-c_{1}}{1.1 k \epsilon^{N}}=\frac{-c_{2}}{\epsilon^{N}} \text { for } \epsilon \in\left(0, \epsilon_{0}\right) .
$$

Integrating $h^{\prime}(t)$ from $T-\epsilon_{0}$ to $T-\epsilon$, we get

$\int_{T-\epsilon_{0}}^{T-\epsilon} h^{\prime}(t) d t \leq \int_{T-\epsilon_{0}}^{T-\epsilon} \frac{-c_{2}}{(T-t)^{N}} d t=\frac{c_{2}}{1-N}\left(\epsilon^{1-N}-\epsilon_{0}^{1-N}\right)\left(\right.$ or $c_{2}\left(\ln \epsilon-\ln \epsilon_{0}\right)$ if $\left.N=1\right)$.

So

$h(T-\epsilon) \leq h\left(T-\epsilon_{0}\right)+\frac{c_{2}}{1-N}\left(\epsilon^{1-N}-\epsilon_{0}^{1-N}\right)\left(\right.$ or $\left.c_{2}\left(\ln \epsilon-\ln \epsilon_{0}\right)\right) \rightarrow-\infty$ as $\epsilon \rightarrow 0$.

This contradicts $h(t)>0$ for $t \in[0, T)$. So there must exist $t_{0} \in(0, T)$ such that $h\left(t_{0}\right)=0$ and $h(t)>0$ for $t \in\left(0, t_{0}\right)$. Integrating the equation from $\epsilon^{\prime}$ to $t_{0}$ and letting $\epsilon^{\prime}$ go to 0 , we get

$$
a\left(t_{0}\right) h^{\prime}\left(t_{0}\right)=-\int_{0}^{t_{0}} b(t) h(t) d t:=c_{3}<0 .
$$

So $h^{\prime}\left(t_{0}\right)=\frac{-c_{3}}{a\left(t_{0}\right)}<0$.

Example $1\left(n=4, \operatorname{Ridge}(u)=S^{1}\right)$. In $S^{3} \subset \mathbb{R}^{4}$, we use coordinates

$$
\begin{cases}x_{1}=\cos \theta \cos \varphi, & x_{2}=\cos \theta \sin \varphi \\ x_{3}=\sin \theta \cos \psi, & x_{4}=\sin \theta \sin \psi,\end{cases}
$$

where $\theta \in\left[0, \frac{\pi}{2}\right]$ and $\varphi, \psi \in[0,2 \pi]$. Note that $\{\theta=0\}=S^{1}(1)$. The Riemannian metric $g$ of $S^{3}$ under this coordinates system reads

$$
\left(\begin{array}{ccc}
1 & 0 & 0 \\
0 & \cos ^{2} \theta & 0 \\
0 & 0 & \sin ^{2} \theta
\end{array}\right)
$$

Assume $u(\theta, \varphi, \psi)=\tau f(\theta)$ for some real number $\tau>0$. Then

$$
\triangle_{S^{3}} u=\frac{1}{\sqrt{\operatorname{det} g}} \partial_{i}\left(\sqrt{\operatorname{det} g} g^{i j} \partial_{j} u\right)=\frac{\tau}{\sin \theta \cos \theta}\left(\sin \theta \cos \theta f^{\prime}\right)^{\prime} .
$$

The equation in (1.1) becomes an ODE

$$
\left(\sin \theta \cos \theta f^{\prime}\right)^{\prime}+3 \sin \theta \cos \theta f=0 .
$$

Now we consider the initial value problem of the second order linear ODE,

$$
\left\{\begin{aligned}
\left(\sin \theta \cos \theta f^{\prime}\right)^{\prime}+3 \sin \theta \cos \theta f & =0, \quad \theta \in\left(0, \frac{\pi}{2}\right) \\
f(0)=1, f^{\prime}(0) & =0 .
\end{aligned}\right.
$$

From Lemma 2.1, we know that there exists $\theta_{0} \in\left(0, \frac{\pi}{2}\right)$ such that $f\left(\theta_{0}\right)=0$, $f^{\prime}\left(\theta_{0}\right)<0$ and $f(\theta)>0$ for $\theta \in\left(0, \theta_{0}\right)$.

Let $u=\frac{-1}{f^{\prime}\left(\theta_{0}\right)} f(\theta)$ for $\theta \in\left[0, \theta_{0}\right)$ and $u \equiv 0$ for $\theta \in\left[\theta_{0}, \frac{\pi}{2}\right]$. We finished the construction of Example 1 
Example $2\left(n=4, \operatorname{Ridge}(u)=S^{1}\left(\frac{1}{\sqrt{2}}\right) \times S^{1}\left(\frac{1}{\sqrt{2}}\right)\right)$. We use the same coordinates as in Example 1 and solve the ODE problem

$$
\left\{\begin{aligned}
\left(\sin \theta \cos \theta f^{\prime}\right)^{\prime}+3 \sin \theta \cos \theta f & =0, \quad \theta \in\left(0, \frac{\pi}{2}\right) \\
f\left(\frac{\pi}{4}\right)=1, f^{\prime}\left(\frac{\pi}{4}\right) & =0 .
\end{aligned}\right.
$$

It is easy to verify that $f(\theta)=f\left(\frac{\pi}{2}-\theta\right)$. From Lemma 2.1, we know there exists $\theta_{0} \in\left(0, \frac{\pi}{4}\right)$ such that $f\left(\theta_{0}\right)=0, f^{\prime}\left(\theta_{0}\right)>0$ and $f(\theta)>0$ for $\theta \in\left(\theta_{0}, \frac{\pi}{2}-\theta_{0}\right)$.

Let $u=\frac{1}{f^{\prime}\left(\theta_{0}\right)} f(\theta)$ for $\theta \in\left(\theta_{0}, \frac{\pi}{2}-\theta_{0}\right)$ and $u \equiv 0$ elsewhere.

Example $3\left(n=5, \operatorname{Ridge}(u)=S^{1}\right)$. In $S^{4} \subset \mathbb{R}^{5}$, we use coordinates

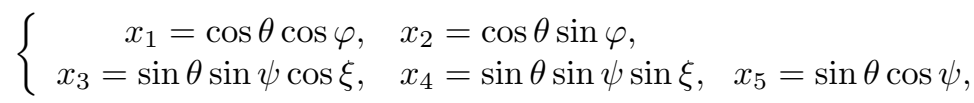

where $\theta \in\left[0, \frac{\pi}{2}\right], \psi \in[0, \pi]$ and $\varphi, \xi \in[0,2 \pi]$. The Riemannian metric $g_{i j}$ of $S^{n-1}$ under this coordinates system $(\theta, \varphi, \psi, \xi)$ reads

$$
\left(\begin{array}{cccc}
1 & 0 & 0 & 0 \\
0 & \cos ^{2} \theta & 0 & 0 \\
0 & 0 & \sin ^{2} \theta & 0 \\
0 & 0 & 0 & \sin ^{2} \theta \sin ^{2} \psi
\end{array}\right) .
$$

Assume $u(\theta, \varphi, \psi)=\tau f(\theta)$. Then

$$
\triangle_{S^{3}} u=\frac{\tau}{\sin ^{2} \theta \cos \theta}\left(\sin ^{2} \theta \cos \theta f^{\prime}\right)^{\prime} .
$$

We solve the ODE problem

$$
\left\{\begin{aligned}
\left(\sin ^{2} \theta \cos \theta f^{\prime}\right)^{\prime}+4 \sin ^{2} \theta \cos \theta f & =0, \quad \theta \in\left(0, \frac{\pi}{2}\right), \\
f(0)=1, f^{\prime}(0) & =0 .
\end{aligned}\right.
$$

From Lemma 2.1, there exists $\theta_{0} \in\left(0, \frac{\pi}{2}\right)$ such that $f\left(\theta_{0}\right)=0, f^{\prime}\left(\theta_{0}\right)<0$ and $f(\theta)>0$ for $\theta \in\left(0, \theta_{0}\right)$.

Let $u=\frac{-1}{f^{\prime}\left(\theta_{0}\right)} f(\theta)$ for $\theta \in\left[0, \theta_{0}\right)$ and $u \equiv 0$ elsewhere.

Example $4\left(n=5, \operatorname{Ridge}(u)=S^{2}\right)$. We use the same coordinates as in Example 3 and solve the ODE problem

$$
\left\{\begin{aligned}
\left(\sin ^{2} \theta \cos \theta f^{\prime}\right)^{\prime}+4 \sin ^{2} \theta \cos \theta f & =0, \quad \theta \in\left(0, \frac{\pi}{2}\right), \\
f\left(\frac{\pi}{2}\right)=1, f^{\prime}\left(\frac{\pi}{2}\right) & =0 .
\end{aligned}\right.
$$

From Lemma 2.1, there exists $\theta_{0} \in\left(0, \frac{\pi}{2}\right)$ such that $f\left(\theta_{0}\right)=0, f^{\prime}\left(\theta_{0}\right)>0$ and $f(\theta)>0$ for $\theta \in\left(\theta_{0}, \frac{\pi}{2}\right)$.

Let $u=\frac{1}{f^{\prime}\left(\theta_{0}\right)} f(\theta)$ for $\theta \in\left(\theta_{0}, \frac{\pi}{2}\right]$ and $u \equiv 0$ elsewhere.

Example $5\left(n=6, \operatorname{Ridge}(u)=S^{1}\right)$. Choose coordinates such that $x_{1}^{2}+x_{2}^{2}=\cos ^{2} \theta$ and $x_{3}^{2}+\cdots+x_{6}^{2}=\sin ^{2} \theta$ with $\theta \in\left[0, \frac{\pi}{2}\right]$. We solve the ODE problem

$$
\left\{\begin{aligned}
\left(\sin ^{3} \theta \cos \theta f^{\prime}\right)^{\prime}+5 \sin ^{3} \theta \cos \theta f & =0, \quad \theta \in\left(0, \frac{\pi}{2}\right), \\
f(0)=1, f^{\prime}(0) & =0 .
\end{aligned}\right.
$$

The rest is the same as Example 1.

Example $6\left(n=6, \operatorname{Ridge}(u)=S^{3}\right)$. With the same coordinates as Example 5, we solve

$$
\left\{\begin{aligned}
\left(\sin ^{3} \theta \cos \theta f^{\prime}\right)^{\prime}+5 \sin ^{3} \theta \cos \theta f & =0, \quad \theta \in\left(0, \frac{\pi}{2}\right), \\
f\left(\frac{\pi}{2}\right)=1, f^{\prime}\left(\frac{\pi}{2}\right) & =0 .
\end{aligned}\right.
$$


The rest is the same as Example 4 .

Example $7\left(n=6, \operatorname{Ridge}(u)=S^{2}\right)$. Choose coordinates such that $x_{1}^{2}+x_{2}^{2}+x_{3}^{2}=$ $\cos ^{2} \theta$ and $x_{4}^{2}+x_{5}^{2}+x_{6}^{2}=\sin ^{2} \theta$ with $\theta \in\left[0, \frac{\pi}{2}\right]$. We solve the ODE problem

$$
\left\{\begin{aligned}
\left(\sin ^{2} \theta \cos ^{2} \theta f^{\prime}\right)^{\prime}+5 \sin ^{2} \theta \cos ^{2} \theta f & =0, \quad \theta \in\left(0, \frac{\pi}{2}\right), \\
f(0)=1, f^{\prime}(0) & =0 .
\end{aligned}\right.
$$

The rest is the same as Example 1 .

Example $8\left(n=6, \operatorname{Ridge}(u)=S^{2}\left(\frac{1}{\sqrt{2}}\right) \times S^{2}\left(\frac{1}{\sqrt{2}}\right)\right)$. With the same coordinates as Example 5, we solve

$$
\left\{\begin{aligned}
\left(\sin ^{2} \theta \cos ^{2} \theta f^{\prime}\right)^{\prime}+5 \sin ^{2} \theta \cos ^{2} \theta f & =0, \quad \theta \in\left(0, \frac{\pi}{2}\right), \\
f\left(\frac{\pi}{4}\right)=1, f^{\prime}\left(\frac{\pi}{4}\right) & =0 .
\end{aligned}\right.
$$

The rest is the same as Example 2 .

Example $9\left(n=7, \operatorname{Ridge}(u)=S^{1}\right)$. Solve the ODE

$$
\left\{\begin{aligned}
\left(\sin ^{4} \theta \cos \theta f^{\prime}\right)^{\prime}+6 \sin ^{4} \theta \cos \theta f & =0, \quad \theta \in\left(0, \frac{\pi}{2}\right), \\
f(0)=1, f^{\prime}(0) & =0 .
\end{aligned}\right.
$$

The rest is the same as Example 1

General case I: Ridge $(u)=S^{p} 1$.

We should solve ODE

$$
\left\{\begin{aligned}
\left(\sin ^{n-2-p} \theta \cos ^{p} \theta f^{\prime}\right)^{\prime}+(n-1) \sin ^{n-2-p} \theta \cos ^{p} \theta f=0, \quad \theta \in\left(0, \frac{\pi}{2}\right), \\
f(0)=1, f^{\prime}(0)=0 .
\end{aligned}\right.
$$

General case II: $\operatorname{Ridge}(u)=S^{\frac{n-2}{2}}\left(\frac{1}{\sqrt{2}}\right) \times S^{\frac{n-2}{2}}\left(\frac{1}{\sqrt{2}}\right) 1$.

We should solve ODE

$$
\left\{\begin{aligned}
\left(\sin ^{\frac{n-2}{2}} \theta \cos ^{\frac{n-2}{2}} \theta f^{\prime}\right)^{\prime}+(n-1) \sin \frac{n-2}{2} \theta \cos ^{\frac{n-2}{2}} \theta f=0, \quad \theta \in\left(0, \frac{\pi}{2}\right), \\
f\left(\frac{\pi}{4}\right)=1, f^{\prime}\left(\frac{\pi}{4}\right)=0 .
\end{aligned}\right.
$$

In both cases, for all $n$ and $p$ Lemma 2.1 guarantees that we can get the expected homogeneous solutions $u$ from the ODE's solutions $f$.

\section{Stability}

Definition 3.1. Let $u \in C\left(\mathbb{R}^{n}\right)$ be a homogeneous solution as in Definition 1.1. We say $u$ is a global minimizer (or an absolute minimum) if for any ball $B \subset \mathbb{R}^{n}$ and any continuous function $v$ defined on $\bar{B}$ such that $v=u$ on $\partial B, J(u, B) \leq J(v, B)$. We say $u$ is stable if for all non-negative $\eta \in C_{0}^{\infty}\left(\mathbb{R}^{n}\right),\left.\frac{d^{2}}{d t^{2}}\right|_{t=0} J\left((u-t \eta)^{+}, B\right) \geq 0$, where $B$ is any ball that contains the supporting set of $\eta$.

In 2], Caffarelli, Jerison and Kenig deduced a necessary condition for stability for the axis-symmetric solutions. However, this result also obviously fits our examples.

Theorem 3.2. Let $u$ be a homogeneous solution as in Definition 1.1. Assume that $\left.u\right|_{S^{n-1}}(x)=f(\theta)$, where $\theta=\operatorname{dist}_{S^{n-1}}\left(x, \operatorname{Ridge}\left(\left.u\right|_{S^{n-1}}\right)\right), \partial \Omega=\left\{\theta=\theta_{0}\right\}$ has constant mean curvature $H_{1}$, and $W(\theta) \in C^{2}\left(0, \theta_{0}\right) \cap C^{1}\left[0, \theta_{0}\right)$ is a function such that $G(x)=r^{-\frac{n-2}{2}} W(\theta)$ is harmonic in $\Gamma$. If $u$ is stable, then

$$
H_{1} \leq\left|W^{\prime}\left(\theta_{0}\right) / W\left(\theta_{0}\right)\right| \text {. }
$$

Proof. See Lemma 1 and Section 3 in [2]. 
For our type I examples, $W(\theta)$ solves the ODE problem

$$
\left\{\begin{aligned}
\left(\sin ^{n-2-p} \theta \cos ^{p} \theta W^{\prime}\right)^{\prime}-\left(\frac{n-2}{2}\right)^{2} \sin ^{n-2-p} \theta \cos ^{p} \theta W=0, \quad \theta \in\left(0, \frac{\pi}{2}\right), \\
W(0)=1, W^{\prime}(0)=0,
\end{aligned}\right.
$$

while

$$
H_{1}=p \tan \theta_{0}-(n-2-p) \cot \theta_{0} .
$$

For the type II examples, $W(\theta)$ solves the ODE problem

$$
\left\{\begin{aligned}
\left(\sin ^{\frac{n-2}{2}} \theta \cos ^{\frac{n-2}{2}} \theta W^{\prime}\right)^{\prime}-\left(\frac{n-2}{2}\right)^{2} \sin ^{\frac{n-2}{2}} \theta \cos ^{\frac{n-2}{2}} \theta W=0, \quad \theta \in\left(0, \frac{\pi}{2}\right), \\
W\left(\frac{\pi}{4}\right)=1, W^{\prime}\left(\frac{\pi}{4}\right)=0,
\end{aligned}\right.
$$

and

$$
H_{1}=\frac{n-2}{2} \cot \theta_{0}-\frac{n-2}{2} \tan \theta_{0} .
$$

All the ODE problems for $f$ and $W$ can be solved by hypergeometric functions using Mathematica. For example, the solution for (2.1) is

$$
f(\theta)=\operatorname{Legendre} P\left[\frac{1}{2}, \cos 2 \theta\right][1]
$$

However, many others have much more complicated expressions. We do not present them in this paper. We computed the values of $\theta_{0}, H_{1}$ and $\left|W^{\prime}\left(\theta_{0}\right) / W\left(\theta_{0}\right)\right|$ numerically using Mathematica:

Example 1: $\theta_{0}=1.14066, H_{1}=1.72088,\left|W^{\prime}\left(\theta_{0}\right) / W\left(\theta_{0}\right)\right|=0.86044, n=4$.

Example 2: $\theta_{0}=0.11103, H_{1}=8.85835,\left|W^{\prime}\left(\theta_{0}\right) / W\left(\theta_{0}\right)\right|=1.69548, n=4$.

Example 3: $\theta_{0}=1.21570, H_{1}=1.95514,\left|W^{\prime}\left(\theta_{0}\right) / W\left(\theta_{0}\right)\right|=1.42123, n=5$.

Example 4: $\theta_{0}=0.61548, H_{1}=2.12132,\left|W^{\prime}\left(\theta_{0}\right) / W\left(\theta_{0}\right)\right|=1.53979, n=5$.

Example 5: $\theta_{0}=1.26177, H_{1}=2.17450,\left|W^{\prime}\left(\theta_{0}\right) / W\left(\theta_{0}\right)\right|=1.99301, n=6$.

Example 6: $\theta_{0}=0.73083, H_{1}=2.45029,\left|W^{\prime}\left(\theta_{0}\right) / W\left(\theta_{0}\right)\right|=2.23113, n=6$.

Example 7: $\theta_{0}=1.04720, H_{1}=2.30940,\left|W^{\prime}\left(\theta_{0}\right) / W\left(\theta_{0}\right)\right|=2.10964, n=6$.

Example 8: $\theta_{0}=0.26180, H_{1}=6.92820,\left|W^{\prime}\left(\theta_{0}\right) / W\left(\theta_{0}\right)\right|=3.46410, n=6$.

Example 9: $\theta_{0}=1.29365, H_{1}=2.37747,\left|W^{\prime}\left(\theta_{0}\right) / W\left(\theta_{0}\right)\right|=2.56697, n=7$.

So the first 8 examples are unstable. Example 9 satisfies the necessary condition for stability. We will check whether it is a global minimizer by the method of De Silva-Jerison [3] in another work. The first type II example that satisfies the condition $H_{1} \leq\left|W^{\prime}\left(\theta_{0}\right) / W\left(\theta_{0}\right)\right|$ appears at 14 dimensions, where $\theta_{0}=0.46189$, $H_{1}=9.06622$ and $\left|W^{\prime}\left(\theta_{0}\right) / W\left(\theta_{0}\right)\right|=9.78353$.

\section{Final REMARK}

The classical result of Serrin [5] confirms that if for some smooth bounded domain $\Omega \subset \mathbb{R}^{n}$, the overdetermined problem

$$
\left\{\begin{aligned}
\triangle u+\lambda u=0, & \text { in } \Omega \\
u=0, \quad \frac{\partial u}{\partial \nu}=-1, & \text { on } \partial \Omega
\end{aligned}\right.
$$

has a positive solution, then $\Omega$ must be a ball and $u$ is radial symmetric. So for the similar problem in a sphere we conjecture that $u$ must be ridge symmetric, which means $u(x)$ only depend on $\operatorname{dist}(x, \operatorname{Ridge}(u)$ ) (or maybe a signed distance function in the case that Ridge $(u)$ is a hypersurface), and all the level sets of $u$ are constant mean curvature surfaces (or even better, for example, isoparametric surfaces).

\footnotetext{
${ }^{1}$ LegendreP $\left[\frac{1}{2}, x\right]=P_{\frac{1}{2}}^{0}(x)$ is the fractional order Legendre function; see http://en.wikipedia. org/wiki/Associated_Legendre_polynomial.
} 
The proof in 2] of the fact that in 3 dimensions all singular homogeneous solutions are unstable depended heavily on the simple geometry of $S^{2}$ and cannot be extended to higher dimensions definitely. Our thought is that one has to investigate sufficiently the symmetry of homogeneous solutions before finding a way to prove the full regularity in dimensions 4-6.

\section{ACKNOWLEDGMENT}

The initial idea in this paper came to mind when the author was a postdoctoral fellow in MSRI for the program: free boundary problems in the spring semester of 2011. The author wants to thank the organizers of the program for providing this opportunity. He also thanks Professor Lizhou Wang and Professor Yu Yuan for helpful discussions.

\section{REFERENCES}

[1] H. W. Alt and L. A. Caffarelli, Existence and regularity for a minimum problem with free boundary, J. Reine Angew. Math. 325 (1981), 105-144. MR618549 (83a:49011)

[2] Luis A. Caffarelli, David Jerison, and Carlos E. Kenig, Global energy minimizers for free boundary problems and full regularity in three dimensions, Noncompact problems at the intersection of geometry, analysis, and topology, Contemp. Math., vol. 350, Amer. Math. Soc., Providence, RI, 2004, pp. 83-97, DOI 10.1090/conm/350/06339. MR2082392(2005e:35258)

[3] Daniela De Silva and David Jerison, A singular energy minimizing free boundary, J. Reine Angew. Math. 635 (2009), 1-21, DOI 10.1515/CRELLE.2009.074. MR2572253 (2010k:49003)

[4] Georg Sebastian Weiss, Partial regularity for a minimum problem with free boundary, J. Geom. Anal. 9 (1999), no. 2, 317-326, DOI 10.1007/BF02921941. MR.1759450(2001b:49053)

[5] James Serrin, A symmetry problem in potential theory, Arch. Rational Mech. Anal. 43 (1971), 304-318. MR0333220 (48 \#11545)

School of Mathematics and Statistics, Xi'an Jiaotong University, Xi'an, People's Republic of China 710049

E-mail address: ghhongmath@mail.xjtu.edu.cn 